\title{
Article
}

\section{On an Anti-Torqued Vector Field on Riemannian Manifolds}

\author{
Sharief Deshmukh ${ }^{1, *(\mathbb{D}, \text { Ibrahim Al-Dayel }}{ }^{2}\left[\right.$ and Devaraja Mallesha Naik ${ }^{3}(\mathbb{C}$ \\ 1 Department of Mathematics, College of Science, King Saud University, P.O. Box 2455, \\ Riyadh 11451, Saudi Arabia \\ 2 Department of Mathematics and Statistics, College of Science, Imam Mohammad Ibn Saud Islamic University, \\ P.O. Box 65892, Riyadh 11566, Saudi Arabia; iaaldayel@imamu.edu.sa \\ 3 Department of Mathematics, CHRIST (Deemed to Be University), Bengaluru 560029, India; \\ devaraja.mallesha@christuniversity.in \\ * Correspondence: shariefd@ksu.edu.sa
}

Citation: Deshmukh, S.; Al-Dayel, I.; Naik, D.M. On an Anti-Torqued Vector Field on Riemannian Manifolds. Mathematics 2021, 9, 2201. https://doi.org/10.3390/ math9182201

Academic Editor: Marian Ioan Munteanu

Received: 29 July 2021

Accepted: 25 August 2021

Published: 8 September 2021

Publisher's Note: MDPI stays neutral with regard to jurisdictional claims in published maps and institutional affiliations.

\begin{abstract}
A torqued vector field $\xi$ is a torse-forming vector field on a Riemannian manifold that is orthogonal to the dual vector field of the 1-form in the definition of torse-forming vector field. In this paper, we introduce an anti-torqued vector field which is opposite to torqued vector field in the sense it is parallel to the dual vector field to the 1-form in the definition of torse-forming vector fields. It is interesting to note that anti-torqued vector fields do not reduce to concircular vector fields nor to Killing vector fields and thus, give a unique class among the classes of special vector fields on Riemannian manifolds. These vector fields do not exist on compact and simply connected Riemannian manifolds. We use anti-torqued vector fields to find two characterizations of Euclidean spaces. Furthermore, a characterization of an Einstein manifold is obtained using the combination of a torqued vector field and Fischer-Marsden equation. We also find a condition under which the scalar curvature of a compact Riemannian manifold admitting an anti-torqued vector field is strictly negative.
\end{abstract}

Keywords: torse-forming vector fields; concircular vector fields; torqued vector fields; Einstein manifolds; scalar curvature; Fischer-Marsden equation

\section{Introduction}

A concircular vector field $\mathbf{w}$ on a Riemannian manifold $(M, g)$ is defined by the equation

$$
\nabla_{E} \mathbf{w}=f E, \quad E \in \mathfrak{X}(M),
$$

where $\nabla$ denotes the Riemannian connection of $(M, g), f$ is a smooth function, and $\mathfrak{X}(M)$ is the Lie algebra of smooth vector fields on $M$. The function $f$ in Equation (1) is called the potential function of $\mathbf{w}$ (cf. [1-5]). Concircular vector fields are well known for their applications in physics (cf. [6-10])

Yano generalized concircular vector fields by torse-forming vector fields (cf. [11]). A vector field $\mathbf{w}$ on $(M, g)$ is a torse-forming vector field if

$$
\nabla_{E} \mathbf{w}=f E+\omega(E) \mathbf{w}, \quad E \in \mathfrak{X}(M),
$$

for a 1-form $\omega$ on $M$. Torse-forming vector fields play a role in physics (cf. $[9,12-18])$. Chen, in [19], considered a specific torse-forming vector field called a torqued vector field. A vector field $\xi$ on $(M, g)$ is said to be a torqued vector field if

$$
\nabla_{E} \xi=\rho E+\omega(E) \xi, \quad \text { and } \quad \omega(\xi)=0, \quad E \in \mathfrak{X}(M)
$$


The 1-form $\omega$ and the function $\rho$ in Equation (3) are called the torqued form and torqued function of the torqued vector field $\xi$ (cf. [19]). If $\omega=0$, then a torqued vector field becomes a concircular vector field. It is interesting to note that the twisted product $I \times{ }_{f} N$ of an interval $I$ and $N$ an $(m-1)$-dimensional Riemannian manifold has a torqued vector field it is not a concircular vector field (cf. [20]). Torqued vector fields severely restrict the geometry of a manifold on which they are defined (cf. [21]).

In present paper, we study a torse-forming vector field on a Riemannian manifold $(M, g)$ for which the vector field $\mathbf{v}$ dual to $\omega(\omega(E)=g(\mathbf{v}, E))$ is parallel to $\mathbf{w}$ (see Equation (2)) as opposed to torqued vector fields (where $\mathbf{v}$ is orthogonal to $\mathbf{w}$ ). In particular, we are interested in a unit torse-forming $\mathbf{w}$ on $(M, g)$ with dual 1-form $\eta$ satisfying $f \eta(E)=-\omega(E)$, that is, $\mathbf{v}=-f \mathbf{w}$ (w is parallel to $\mathbf{v}$ ) and call this torse-forming vector field an anti-torqued vector field on the Riemannian manifold $(M, g)$. Thus, for an anti-torqued vector field $\mathbf{w}$, we have

$$
\nabla_{E} \mathbf{w}=f(E-\eta(E) \mathbf{w}), \quad E \in \mathfrak{X}(M),
$$

where $\eta$ is dual to unit anti-torqued vector field $\mathbf{w}$ and $f$ is a nonzero smooth function defined on $M$. We call $f$ the potential function of the anti-torqued vector field $\mathbf{w}$. Note that $f$ is nonzero does not mean it is nowhere zero, in fact there is no open subset of $M$ on which potential function $f$ is zero. We require potential function to be nonzero so that the anti-torqued vector field is not parallel. From the definition of an anti-torqued vector field, it follows that, it is that torse-forming vector field, which under no condition is a concircular vector field nor a Killing vector field. Thus, an anti-torqued vector field will have a unique status among the special vector fields.

Note that if $V$ is a nowhere vanishing vector field on a Riemannian manifold $(M, g)$ that satisfies

$$
\nabla_{E} V=\sigma(E-\beta(E) V), \quad E \in \mathfrak{X}(M),
$$

where $\beta(E)=g(V, E)$ the dual to $V$, then defining $\mathbf{w}=\|V\|^{-1} V$, we get the unit vector field $\mathbf{w}$ satisfying

$$
\begin{aligned}
\nabla_{E} \mathbf{w} & =-\frac{1}{\|V\|^{2}} E(\|V\|) V+\frac{\sigma}{\|V\|}(E-\beta(E) V) \\
& =-\frac{1}{2\|V\|^{3}} E(g(V, V)) V+\frac{\sigma}{\|V\|}(E-\beta(E) V) \\
& =-\frac{1}{\|V\|^{3}}\left(g\left(\nabla_{E} V, V\right)\right) V+\frac{\sigma}{\|V\|}(E-\beta(E) V) \\
& =\frac{\sigma}{\|V\|}\left(E-g\left(E, \frac{V}{\|V\|}\right) \frac{V}{\|V\|}\right) \\
& =f(E-\eta(E) \mathbf{w}),
\end{aligned}
$$

where $\sigma=f\|V\|$ and $\eta(E)=g(E, \mathbf{w})$ and we have used Equation (5). Thus, nowhere zero torse-forming $V$ satisfying (5) reduces to an anti-torqued vector field $\mathbf{w}$ on $(M, g)$ with $f=\sigma\|V\|^{-1}$.

It is observed that on a compact and simply connected Riemannian manifold there is no anti-torqued vector field (see Example 1, in next section). Furthermore, unlike torqued vector fields, an anti-torqued vector field under no circumstances can be reduced to a concircular vector field or a Killing vector field. Thus, anti-torqued vector fields form an independent sub-class of torse-forming vector fields among the classes of special vector fields. It is a known fact that torse-forming vector fields have immense applications 
in physics-an anti-torqued vector field being a particular form of torse-forming vector fields will have many applications to physical sciences. In present paper, we use an antitorqued vector field to find two characterizations of Euclidean spaces (see Corollary 1 and Theorem 2). One of most important spaces in geometry is an Einstein manifold and therefore the most interesting question in geometry is to find different characterizations of Einstein manifolds. We use an anti-torqued vector field in finding a characterization of an Einstein manifold (see Theorem 3). We also find a condition under which the scalar curvature of a compact Riemannian manifold admitting an anti-torqued vector field is strictly negative (see Theorem 4).

\section{Preliminaries}

Given a smooth function $\varphi$ on a Riemannian manifold $(M, g)$, the Hessian operator $H^{\varphi}$ of $\varphi$ is

$$
H^{\varphi}(E)=\nabla_{E} \operatorname{grad} \varphi, \quad E \in \mathfrak{X}(M),
$$

where $\operatorname{grad} \varphi$ is the gradient of $\varphi$ and the Hessian of $\varphi$ is

$$
\operatorname{Hess}(\varphi)(E, F)=g\left(H^{\varphi}(E), F\right), \quad E, F \in \mathfrak{X}(M) .
$$

The Laplace operator $\Delta$ on $(M, g)$ is given by

$$
\Delta \varphi=\operatorname{div}(\operatorname{grad} \varphi),
$$

and we also have

$$
\Delta \varphi=\operatorname{tr} H^{\varphi} .
$$

Let $\nabla$ the Levi-Civita connection on an $m$-dimensional Riemannian manifold $(M, g)$. The curvature tensor field $R$ is given by

$$
R\left(E_{1}, E_{2}\right) E_{3}=\left[\nabla_{E_{1}}, \nabla_{E_{2}}\right] E_{3}-\nabla_{\left[E_{1}, E_{2}\right]} E_{3}, E_{1}, E_{2}, E_{3} \in \mathfrak{X}(M) .
$$

The Ricci tensor Ric of $(M, g)$ is

$$
\operatorname{Ric}\left(E_{1}, E_{2}\right)=\sum_{i=1}^{m} g\left(R\left(e_{i}, E_{1}\right) E_{2}, e_{i}\right),
$$

where $\left\{e_{1}, \ldots, e_{m}\right\}$ is a local frame on $M$. The Ricci tensor gives the Ricci operator $Q$ defined by

$$
\operatorname{Ric}\left(E_{1}, E_{2}\right)=g(Q E, F), \quad E_{1}, E_{2} \in \mathfrak{X}(M) .
$$

The scalar curvature $S$ of the Riemannian manifold $(M, g)$ is defined by

$$
S=\operatorname{tr} Q
$$

and it satisfies

$$
\frac{1}{2} \operatorname{grad} S=\sum_{i=1}^{m}(\nabla Q)\left(e_{i}, e_{i}\right),
$$

where we are using the notation $(\nabla Q)\left(E_{1}, E_{2}\right)=\nabla_{E_{1}} Q E_{2}-Q \nabla_{E_{1}} E_{2}$. Now, using the definition of curvature tensor field and the Hessian operator $H^{\varphi}$ it follows that

$$
R\left(E_{1}, E_{2}\right) \operatorname{grad} \varphi=\left(\nabla H^{\varphi}\right)\left(E_{1}, E_{2}\right)-\left(\nabla H^{\varphi}\right)\left(E_{2}, E_{1}\right), \quad E_{1}, E_{2} \in \mathfrak{X}(M) .
$$


Next, we wish to compute the gradient of the function $\Delta \varphi$ and to achieve it we use Equations (7) and (9), a local frame in neighborhood of normal coordinates and proceed as follows

$$
\begin{aligned}
E(\Delta \varphi) & =E\left(\sum_{i=1}^{m} g\left(H^{\varphi} e_{i}, e_{i}\right)\right)=\sum_{i=1}^{m} g\left(\left(\nabla H^{\varphi}\right)\left(E, e_{i}\right), e_{i}\right) \\
& =\sum_{i=1}^{m} g\left(\left(\nabla H^{\varphi}\right)\left(e_{i}, E\right)+R\left(E, e_{i}\right) \operatorname{grad} \varphi, e_{i}\right) \\
& =\sum_{i=1}^{m} g\left(E,\left(\nabla H^{\varphi}\right)\left(e_{i}, e_{i}\right)\right)-\operatorname{Ric}(E, \operatorname{grad} \varphi), \quad E \in \mathfrak{X}(M),
\end{aligned}
$$

where the symmetry of the operator $H^{\varphi}$ is used. Thus, we have

$$
\operatorname{grad}(\Delta \varphi)=-Q(\operatorname{grad} \varphi)+\sum_{i=1}^{m}\left(\nabla H^{\varphi}\right)\left(e_{i}, e_{i}\right) .
$$

Let $\mathbf{w}$ be an anti-torqued vector field on a $(M, g)$. Using the defining Equation (4), we get the following equations

$$
R\left(E_{1}, E_{2}\right) \mathbf{w}=E_{1}(f)\left(E_{2}-\eta\left(E_{2}\right) \mathbf{w}\right)-E_{2}(f)\left(E_{1}-\eta\left(E_{1}\right) \mathbf{w}\right)-f^{2}\left(\eta\left(E_{2}\right) E_{1}-\eta\left(E_{1}\right) E_{2}\right),
$$

where $E_{1}, E_{2} \in \mathfrak{X}(M)$, and the above equation implies

$$
\operatorname{Ric}(E, \mathbf{w})=-(m-2) E(f)-\left(\mathbf{w}(f)+(m-1) f^{2}\right) \eta(E),
$$

that is,

$$
Q(\mathbf{w})=-(m-2) \operatorname{grad} f-\left(\mathbf{w}(f)+(m-1) f^{2}\right) \mathbf{w} .
$$

Furthermore, by Equation (4), we see that $\operatorname{div} \mathbf{w}=(m-1) f$ and that $\operatorname{div}(f \mathbf{w})=\mathbf{w}(f)+$ $(m-1) f^{2}$. Thus, if the anti-torqued $\mathbf{w}$ is defined on a compact $(M, g)$, then

$$
\int_{M} f=0, \quad \int_{M} \mathbf{w}(f)=-(m-1) \int_{M} f^{2} .
$$

Now, we discuss some examples of anti-torqued vector fields as well as about manifolds on which anti-torqued vector fields do not exist.

Example 1. Let $(M, g)$ be a compact and simply connected Riemannian manifold and $\mathbf{w}$ be an anti-torqued vector field on $(M, g)$. Using Equation (4), we observe that $d \eta=0$ and $M$ being simply connected, $\eta=d \varphi$ for a function $\varphi$, which implies, $\mathbf{w}=\operatorname{grad} \varphi$. However, $M$ being compact, the function $\varphi$ has a critical point $x \in M$, where $\mathbf{w}(x)=0$ and this contradicts the fact $\|\mathbf{w}\|=1$. Thus, $(M, g)$ does not admit an anit-torqued vector field. In particular, spheres $\mathbf{S}^{m}(c)$, $m>1$, does not admit an anti-torqued vector field.

Example 2. Let $\mathbf{w}$ be the globally defined unit vector field on the unit circle $\mathbf{S}^{1}$ and $\varphi$ be a positive function on $\mathbf{S}^{1}$. Consider the warped product $M=\mathbf{S}^{1} \times{ }_{\varphi} N$, with warped product metric $g=d \theta^{2}+\varphi^{2} \bar{g}$, where $(N, \bar{g})$ is an $(m-1)$-dimensional Riemannian manifold. Then taking $E=h \mathbf{w}+U, h$ a smooth function on $\mathbf{S}^{1}$ and $U \in \mathfrak{X}(N)$, we get (cf. [22]))

$$
\nabla_{E} \mathbf{w}=\frac{\mathbf{w}(\varphi)}{\varphi} U=\frac{\mathbf{w}(\varphi)}{\varphi}(E-h \mathbf{w})=\frac{\mathbf{w}(\varphi)}{\varphi}(E-g(E, \mathbf{w}) \mathbf{w}) .
$$


Hence, $\mathbf{w}$ is an anti-torqued vector field on $(M, g)$. In particular the compact warped product space $\mathbf{S}^{1} \times{ }_{\varphi} \mathbf{S}^{m-1}$ admits an anti-torqued vector field.

Example 3. Consider the Euclidean space $\mathbf{E}^{m}$. There is a smooth function $\varphi$ on $\mathbf{E}^{m}$ satisfying $\|\operatorname{grad} \varphi\|=1$ and $\varphi \Delta \varphi=m-1$ (cf. [23]). We denote by $g$ the Euclidean metric and by $\nabla$ the Euclidean connection on $\mathbf{E}^{m}$. We have a unit vector field $\mathbf{w}$ on $\mathbf{E}^{m}$ given by $\mathbf{w}=\operatorname{grad} \varphi$ and $a$ nonzero smooth function $f$ given by

$$
\varphi=\frac{1}{f}
$$

Thus, $f \mathbf{w}=-\varphi \operatorname{grad} f$, that is,

$$
\mathbf{w}=-\frac{\operatorname{grad} f}{f^{2}}
$$

and we get

$$
g(\operatorname{grad} f, \operatorname{grad} \varphi)=-f^{2}
$$

Now, for $E \in \mathfrak{X}\left(\mathbf{E}^{m}\right)$ using $\|\operatorname{grad} \varphi\|=1$, we get $g\left(H^{\varphi}(E)\right.$, grad $\left.\varphi\right)=0$. Thus, we have

$$
H^{\varphi}(\operatorname{grad} \varphi)=0
$$

Differentiating above equation with respect to $E$, we get

$$
\left(\nabla H^{\varphi}\right)(E, \operatorname{grad} \varphi)+H^{\varphi}(E)=0 .
$$

Using a local frame $\left\{e_{1}, \ldots, e_{m}\right\}$ and choosing $E=e_{i}$ and on taking the inner product with $e_{i}$ in above equation, on summing, we arrive at

$$
g\left(\operatorname{grad} \varphi, \sum_{i=1}^{m}\left(\nabla H^{\varphi}\right)\left(e_{i}, e_{i}\right)\right)+\left\|H^{\varphi}\right\|^{2}=0
$$

Now, using Equation (10), with $Q=0$ for the Euclidean space $\mathbf{E}^{m}$, in above equation we have

$$
g(\operatorname{grad} \varphi, \operatorname{grad}(\Delta \varphi))+\left\|H^{\varphi}\right\|^{2}=0
$$

We compute

$$
\left\|H^{\varphi}-f I+f \eta \otimes \mathbf{w}\right\|^{2}=\left\|H^{\varphi}\right\|^{2}+m f^{2}+f^{2}-2 f \Delta \varphi+2 f g\left(H^{\varphi}(\mathbf{w}), \mathbf{w}\right)-2 f^{2} .
$$

Using (14) in the form $H^{\varphi}(\mathbf{w})=0$ and Equation (15) with above equation, we get

$$
\left\|H^{\varphi}-f I+f \eta \otimes \mathbf{w}\right\|^{2}=-g(\operatorname{grad} \varphi, \operatorname{grad}(\Delta \varphi))+(m-1) f^{2}-2 f \Delta \varphi .
$$

Note that $\operatorname{grad} \Delta \varphi=\operatorname{grad}(m-1) f=(m-1) \operatorname{grad} f$, and using Equation (13), we get

$$
g(\operatorname{grad} \varphi, \operatorname{grad}(\Delta \varphi))=(m-1) g(\operatorname{grad} \varphi, \operatorname{grad} f)=-(m-1) f^{2} .
$$

Furthermore, we have $f \Delta \varphi=(m-1) f^{2}$. Combining these two outcomes in Equation (16), we conclude

$$
\left\|H^{\varphi}-f I+f \eta \otimes \mathbf{w}\right\|^{2}=(m-1) f^{2}+(m-1) f^{2}-2(m-1) f^{2}=0 .
$$

Hence, $H^{\varphi}-f I+f \eta \otimes \mathbf{w}=0$, that is,

$$
\nabla_{E} \mathbf{w}=f(E-\eta(E) \mathbf{w}), \quad E \in \mathfrak{X}\left(\mathbf{E}^{m}\right),
$$


proving that $\mathbf{w}$ is an anti-torqued vector field.

Example 4. Let $(M, \phi, \xi, \eta, g)$ be a $(2 m+1)$-dimensional $\beta$-Kenmotsu manifold (cf. [13,24]). Then $\xi$ is a unit vector field that satisfies

$$
\nabla_{E} \xi=\beta(E-\eta(E) \xi), \quad E \in \mathfrak{X}(M)
$$

for a smooth function $\beta$. Thus, $\xi$ is an anti-torqued vector field.

Example 5. Consider the connected Riemannian manifold $(M, g)$, where $M=\mathbf{E}^{m}-\{0\}$ and $g$ is Euclidean metric. Define $\mathbf{w}$ on $M$ by

$$
\mathbf{w}=\frac{u}{\|u\|}
$$

where $u$ is position vector on $\mathbf{E}^{m}$. We have

$$
\begin{aligned}
\nabla_{E} \mathbf{w} & =-\frac{1}{\|u\|^{2}} E(\|u\|) u+\frac{1}{\|u\|} E=-\frac{1}{\|u\|^{3}} g(E, u) u+\frac{1}{\|u\|} E \\
& =\frac{1}{\|u\|}(E-g(E, \mathbf{w}) \mathbf{w}), \quad E \in \mathfrak{X}(M) .
\end{aligned}
$$

Thus, $\mathbf{w}$ is an anti-torqued vector field on $(M, g)$.

\section{Characterizing Euclidean Spaces via Anti-Torqued Vector Fields}

In Example 3, we have seen that a smooth function $\varphi$ on the Euclidean space $\mathbf{E}^{m}$ satisfying $\|\operatorname{grad} \varphi\|=1$ and $\varphi \Delta \varphi=m-1$ gives anti-torqued vector field $\mathbf{w}=\operatorname{grad} \varphi$. In this section, we shall show that the converse too holds. Indeed we prove

Theorem 1. If an m-dimensional complete and connected Riemannian manifold $(M, g)$ admits a smooth function $\varphi$ satisfying $\|\operatorname{grad} \varphi\|=1$ and $\varphi \Delta \varphi=m-1$ such that the unit vector field $\mathbf{w}=\operatorname{grad} \varphi$ is an anti-torqued vector field, then $(M, g)$ is isometric to the Euclidean space $\mathbf{E}^{m}$.

Proof. Let $\mathbf{w}=\operatorname{grad} \varphi$ be an anti-torqued vector field on $(M, g)$. Using Equation (4), we get

$$
H^{\varphi}(E)=f(E-\eta(E) \mathbf{w}), \quad E \in \mathfrak{X}(M),
$$

that is, $\Delta \varphi=(m-1) f$, which in view of $\varphi \Delta \varphi=m-1$ implies $f \varphi=1$. Define $h=\varphi^{2}$, which gives gradh $=2 \varphi \mathbf{w}$. Using Equation (4), we get

$$
H^{h}(E)=2 E(\varphi) \mathbf{w}+2 \varphi f(E-\eta(E) \mathbf{w})=2 E(\varphi) \mathbf{w}+2(E-\eta(E) \mathbf{w}) .
$$

Using the fact $\eta(E)=g(E, \mathbf{w})=g(E, \operatorname{grad} \varphi)=E(\varphi)$ in above equation, we conclude

$$
H^{h}(E)=2 E, \quad E \in \mathfrak{X}(M) .
$$

Thus,

$$
\operatorname{Hess}(h)=2 g,
$$

where $h$ is a non-constant function (as $\varphi$ is). Hence, by above equation, we conclude that $(M, g)$ is isometric to the Euclidean space $\mathbf{E}^{m}$ (cf. [25])

Combining Example 3 with above result, we get the following: 
Corollary 1. An m-dimensional complete and connected Riemannian manifold $(M, g)$ admits a smooth function $\varphi$ satisfying $\|\operatorname{grad} \varphi\|=1, \varphi \Delta \varphi=m-1$ and the unit vector field $\mathbf{w}=\operatorname{grad} \varphi$ is an anti-torqued vector field if and only if $(M, g)$ is isometric to the Euclidean space $\mathbf{E}^{m}$.

Note that the Ricci operator $Q=0$ on an Euclidean space $\mathbf{E}^{m}$ and $\mathbf{E}^{m}$ is simply connected space that admits an anti-torqued vector field. One naturally is inclined to know whether a simply connected Ricci flat Riemannian manifold that admits an anti-torqued vector field is necessarily isometric to the Euclidean space. We show that this assertion is true with Ricci flatness replaced by the weaker condition, i.e., the Ricci operator is annihilated by $\mathbf{w}$.

Theorem 2. An m-dimensional simply connected Riemannian manifold $(M, g),(m>2)$, admits an anti-torqued vector field $\mathbf{w}$ with potential function $f$ and that $\mathbf{w}$ annihilates the Ricci operator if and only if $(M, g)$ is isometric to the Euclidean space $\mathbf{E}^{m}$.

Proof. Let $\mathbf{w}$ be an anti-torqued vector field on a simply connected Riemannian manifold $(M, g),(m>2)$, such that $Q(\mathbf{w})=0$. Then Equation (11) implies

$$
(m-2) \operatorname{grad} f+\left(\mathbf{w}(f)+(m-1) f^{2}\right) \mathbf{w}=0 .
$$

Equation (17) on taking the inner product with $\mathbf{w}$, gives

$$
\mathbf{w}(f)=-f^{2}
$$

and combining it with Equation (17), in view of $m>2$, we arrive at

$$
\operatorname{grad} f=\mathbf{w}(f) \mathbf{w} .
$$

Using the definition of anti-torqued vector field, that is, Equation (4), we see that $d \eta=0$ and $M$ being simply connected the closed form $\eta$ is exact. Therefore, there is a function $\varphi$ satisfying $\eta=d \varphi$. Consequently, we have $\mathbf{w}=\operatorname{grad} \varphi$ and combining it with (18) and (19), we conclude

$$
\operatorname{grad} \varphi=-\frac{\operatorname{grad} f}{f^{2}}=\operatorname{grad}\left(\frac{1}{f}\right) .
$$

The above equation implies

$$
\varphi=\frac{1}{f}+c,
$$

for a constant $c$. Taking $\bar{\varphi}=\varphi-c$, we have $\operatorname{grad} \bar{\varphi}=\mathbf{w}$ and $\Delta \bar{\varphi}=\operatorname{div} \mathbf{w}=(m-1) f$ (outcome of Equation (4)). Thus, we have $\bar{\varphi} \Delta \bar{\varphi}=(m-1)$. This proves that the smooth function $\bar{\varphi}$ on $M$ satisfies $\|\operatorname{grad} \bar{\varphi}\|=1, \bar{\varphi} \Delta \bar{\varphi}=(m-1)$ and $\operatorname{grad} \bar{\varphi}$ is an anti-torqued vector field. Hence, by previous result, we get that $(M, g)$ is isometric to $\mathbf{E}^{m}$. Converse is trivial.

\section{A Characterization of Einstein Manifolds}

In this last section, first we use an anti-torqued vector field $\mathbf{w}$ on a Riemannian manifold $(M, g)$ and seek the conditions under which $(M, g)$ is an Einstein manifold. Here we wish to use the Fischer-Marsden differential equation on a Riemannian manifold (cf. [26]). They considered the following differential equation

$$
(\Delta \varphi) g+\varphi \operatorname{Ric}=\operatorname{Hess}(\varphi)
$$


on a connected Riemannian manifold $(M, g)$ and have shown that the Riemannian manifold possessing a non-trivial solution of Equation (20) equation must have a constant scalar curvature. They also conjectured that a compact Riemannian admitting a non-trivial solution of differential Equation (20) must be an Einstein manifold. It is an interesting question to find conditions on a non-compact Riemannian manifold possessing non-trivial solution of Equation (20) to be an Einstein manifold.

Theorem 3. Let $\mathbf{w}$ be an anti-torqued vector field on an m-dimensional connected Riemannian manifold $(M, g),(m>2)$ with potential function $f$ such that $H^{f}(\mathbf{w})=f \mathbf{w}(f) \mathbf{w}$. Then, $(M, g)$ is an Einstein manifold of constant scalar curvature $-m(m-1) \mathbf{w}(f)$ if and only if $\mathbf{w}$ is an eigenvector of the Ricci operator and the potential function $f$ is a non-trivial solution of the FischerMarsden equation.

Proof. Suppose $\mathbf{w}$ is an anti-torqued vector field on a connected Riemannian manifold $(M, g)$ satisfying

$$
H^{f}(\mathbf{w})=f \mathbf{w}(f) \mathbf{w}
$$

and $Q(\mathbf{w})=\delta \mathbf{w}$ for some smooth function $\delta$. Then using Equation (11), we have

$$
\delta \mathbf{w}=-(m-2) \operatorname{grad} f-\left(\mathbf{w}(f)+(m-1) f^{2}\right) \mathbf{w} .
$$

The above equation implies

$$
\delta=-(m-1)\left(\mathbf{w}(f)+f^{2}\right)
$$

and inserting this value of $\delta$ in Equation (21), in view of $m>2$, we get

$$
\operatorname{grad} f=\mathbf{w}(f) \mathbf{w} .
$$

Differentiating Equation (22) with respect to $E$ and using Equation (4), we have

$$
H^{f}(E)=E(\mathbf{w}(f)) \mathbf{w}+f \mathbf{w}(f)(E-\eta(E) \mathbf{w}) .
$$

Note that owing to Equation (4), we see $\nabla_{\mathbf{w}} \mathbf{w}=0$ and therefore,

$$
\operatorname{Hess}(f)(\mathbf{w}, \mathbf{w})=\mathbf{w w}(f) .
$$

Now, taking $E=\mathbf{w}$ in Equation (23) we have

$$
H^{f}(\mathbf{w})=\operatorname{Hess}(f)(\mathbf{w}, \mathbf{w}) \mathbf{w} .
$$

Furthermore, using Equations (21) and (24), we have

$$
\operatorname{Hess}(f)(\mathbf{w}, \mathbf{w})=f \mathbf{w}(f), \quad H^{f}(\mathbf{w})=f \mathbf{w}(f) \mathbf{w} .
$$

We compute $\Delta f$ using Equations (23) and (25) to arrive at

$$
\Delta f=(m-1) f \mathbf{w}(f)+\operatorname{Hess}(f)(\mathbf{w}, \mathbf{w})=m f \mathbf{w}(f) .
$$

We write the Equation (23) as $H^{f}(E)=f \mathbf{w}(f) E+(E \mathbf{w}(f)-f \mathbf{w}(f) \eta(E)) \mathbf{w}$ and compute $\left\|H^{f}\right\|^{2}=m f^{2} \mathbf{w}(f)^{2}+\|\operatorname{grad} \mathbf{w}(f)\|^{2}+f^{2} \mathbf{w}(f)^{2}-2 f \mathbf{w}(f) \mathbf{w} \mathbf{w}(f)+2 f \mathbf{w}(f)(\mathbf{w} \mathbf{w}(f)-f \mathbf{w}(f))$, 
that is,

$$
\left\|H^{f}\right\|^{2}=(m-1) f^{2} \mathbf{w}(f)^{2}+\|\operatorname{grad} \mathbf{w}(f)\|^{2} .
$$

Using this equation and Equation (26) we have

$$
\left\|H^{f}\right\|^{2}-\frac{1}{m}(\Delta f)^{2}=\|\operatorname{grad} \mathbf{w}(f)\|^{2}-f^{2} \mathbf{w}(f)^{2} .
$$

Using Equation (23), we get $E(\mathbf{w}(f))=g\left(H^{f}(E), \mathbf{w}\right)$ and it implies grad $\mathbf{w}(f)=H^{f}(\mathbf{w})$. Equation (25), implies $\operatorname{grad} \mathbf{w}(f)=f \mathbf{w}(f) \mathbf{w}$, that is,

$$
\|\operatorname{grad} \mathbf{w}(f)\|^{2}=f^{2} \mathbf{w}(f)^{2} .
$$

Using Equations (27) and (28), we conclude

$$
\left\|H^{f}\right\|^{2}=\frac{1}{m}(\Delta f)^{2}
$$

The Schwarz's inequality implies $\left\|H^{f}\right\|^{2} \geq \frac{1}{m}(\Delta f)^{2}$, and for the equality to hold $H^{f}=\frac{\Delta f}{m} I$. Therefore, in view of Equation (26), we get

$$
H^{f}=f \mathbf{w}(f) I \text { and } \operatorname{Hess}(f)=f \mathbf{w}(f) g .
$$

Now, the potential function $f$ satisfies Equation (20), that is,

$$
(\Delta f) g+f \operatorname{Ric}=\operatorname{Hess}(f)
$$

and above equation together with Equations (26) and (29) implies

$$
f[\mathbf{w}(f)(m-1) g+R i c]=0 .
$$

Since, $f$ is a non-trivial solution of Equation (20), $f \neq 0$ and on connected $M$ above equation yields

$$
\text { Ric }=-\mathbf{w}(f)(m-1) g .
$$

Hence, as $m \geq 3,(M, g)$ is an Einstein manifold of constant scalar curvature

$$
S=-m(m-1) \mathbf{w}(f)
$$

Conversely, suppose $(M, g)$ is an Einstein manifold of constant scalar curvature $-m(m-1) \mathbf{w}(f)$ that admits an anti-torqued vector field $\mathbf{w}$ that satisfies $H^{f}(\mathbf{w})=f \mathbf{w}(f) \mathbf{w}$. Since $(M, g)$ is Einstein, we have $Q \mathbf{w}=\delta \mathbf{w}$ for a constant $\delta$, and following argument similar to before Equation (22), we see Equation (22) holds. Thus, Equations (22)-(26) hold. Thus, on taking the inner product with $E_{1} \in \mathfrak{X}(M)$ in (23), we get

$$
\operatorname{Hess}(f)\left(E, E_{1}\right)=E(\mathbf{w}(f)) \eta\left(E_{1}\right)+f \mathbf{w}(f) g\left(E, E_{1}\right)-f \mathbf{w}(f) \eta(E) \eta\left(E_{1}\right) .
$$

Using symmetry of $\operatorname{Hess}(f)$ in (30), we have $E(\mathbf{w}(f)) \eta\left(E_{1}\right)=E_{1}(\mathbf{w}(f)) \eta(E)$ and using $E_{1}=\mathbf{w}$, we obtain $E(\mathbf{w}(f))=\mathbf{w}(\mathbf{w}(f)) \eta(E)=\operatorname{Hess}(f)(\mathbf{w}, \mathbf{w}) \eta(E)$. Inserting Equation (25) in this last equation, we arrive at

$$
E(\mathbf{w}(f))=f \mathbf{w}(f) \eta(E) .
$$


Consequently, Equation (30) implies

$$
\operatorname{Hess}(f)\left(E, E_{1}\right)=f \mathbf{w}(f) g\left(E, E_{1}\right), \quad E, E_{1} \in \mathfrak{X}(M) .
$$

Finally, using Equation (26) and Ric $=-(m-1) \mathbf{w}(f) g$, we have

$$
(\Delta f) g+f R i c=m f \mathbf{w}(f)-(m-1) f \mathbf{w}(f)=f \mathbf{w}(f)
$$

and using (31) with above equation, we conclude that function $f$ satisfies the equation

$$
(\Delta f) g+f \operatorname{Ric}=\operatorname{Hess}(f),
$$

which is Fischer-Marsden equation.

Since, we did not discuss the role of an anti-torqued vector field on a compact manifolds (except in Example 2), we shall now describe the relationship between the support function $f$ of an anti-torqued vector field $\mathbf{w}$ on a compact Riemannian manifold and the scalar curvature $S$ as well as the Ricci curvature along the anti-torqued vector field. We prove that if the scalar curvature satisfies some pinching condition, then $S$ is negative.

Theorem 4. Let $\mathbf{w}$ be an anti-torqued vector field with potential function $f$ on an m-dimensional compact and connected Riemannian manifold $(M, g), m>3$. If the scalar curvature $S$ of $(M, g)$ satisfies $f S+(m-1) f^{3} \leq 0$, then the scalar curvature is given by $S=-(m-1) f^{2}$.

Proof. Note that using Equation (4), we have

$$
\operatorname{div}(S \mathbf{w})=\mathbf{w}(S)+(m-1) f S .
$$

Choosing a local orthonormal frame $\left\{e_{1}, \ldots, e_{m}\right\}$, we compute

$$
\operatorname{div} Q(\mathbf{w})=\sum_{i=1}^{m}\left[g\left((\nabla Q)\left(e_{i}, \mathbf{w}\right)+Q\left(\nabla_{e_{i}} \mathbf{w}\right), e_{i}\right)\right],
$$

which on using equation symmetry of $Q$ and Equations (4) and (8), gives

$$
\operatorname{div} Q(\mathbf{w})=\frac{1}{2} \mathbf{w}(S)+f S-f \operatorname{Ric}(\mathbf{w}, \mathbf{w}) .
$$

Using (32) in the integral of the above equation, we have

$$
\int_{M}\left[\frac{1}{2}(m-3) f S+f \operatorname{Ric}(\mathbf{w}, \mathbf{w})\right]=0 .
$$

Using Equation (11), we have $\operatorname{Ric}(\mathbf{w}, \mathbf{w})=-(m-1)\left(\mathbf{w}(f)+f^{2}\right)$. Inserting this value in Equation (34), we get

$$
\int_{M}\left[\frac{1}{2}(m-3) f S-\frac{(m-1)}{2} \mathbf{w}\left(f^{2}\right)-(m-1) f^{3}\right]=0 .
$$

Note that $\operatorname{div}\left(f^{2} \mathbf{w}\right)=\mathbf{w}\left(f^{2}\right)+(m-1) f^{3}$ and using this expression in above integral, we arrive at

$$
(m-3) \int_{M}\left[f S+(m-1) f^{3}\right]=0 .
$$


As, $m>3$ and $f S+(m-1) f^{3} \leq 0$ above integral implies $f\left(S+(m-1) f^{2}\right)=0$. However, as the potential function $f \neq 0$ and $M$ is connected, we get $S$ is given by $S=-(m-1) f^{2}$.

An anti-torqued vector field restricts the behavior of the potential function and the Ricci curvature of a 3-dimensional compact Riemannian manifold as seen the following:

Corollary 2. Let $\mathbf{w}$ be an anti-torqued vector field with potential function $f$ on a 3-dimensional compact and connected Riemannian manifold $(M, g)$. Then the potential function $f$ and the Ricci curvature $\operatorname{Ric}(\mathbf{w}, \mathbf{w})$ do not have same sign throughout $M$.

Proof. Suppose that $f$ and $\operatorname{Ric}(\mathbf{w}, \mathbf{w})$ have same sign throughout $M$. Then we have $f \operatorname{Ric}(\mathbf{w}, \mathbf{w}) \geq 0$. Using this information and $m=3$ in Equation (34), we conclude

$$
f \operatorname{Ric}(\mathbf{w}, \mathbf{w})=0 .
$$

However, the potential function $f \neq 0$ and $M$ is connected, we get $\operatorname{Ric}(\mathbf{w}, \mathbf{w})=0$. Thus, using Equation (11), we have $\mathbf{w}(f)=-f^{2}$. Inserting this equation in (12) with $m=3$, we conclude

$$
\int_{M} f^{2}=0
$$

that is, $f=0$ a contradiction.

Author Contributions: Conceptualization and methodology, S.D., I.A.-D., D.M.N.; formal analysis, I.A.-D.; writing original draft preparation, S.D., I.A.-D., D.M.N.; writing review and editing, S.D. and D.M.N.; supervision, S.D.; project administration I.A.-D. All authors have read and agreed to the published version of the manuscript.

Funding: The authors extend their appreciation to the Deanship of Scientific Research at Imam Mohammad Ibn Saud Islamic University for funding this through Research Group no. RG-21-09-09.

Institutional Review Board Statement: Not applicable.

Informed Consent Statement: Not applicable.

Data Availability Statement: Not applicable.

Conflicts of Interest: The authors declare no conflict of interest.

\section{References}

1. Chen, B.-Y. Some results on concircular vector fields and their applications to Ricci solitons. Bull. Korean Math. Soc. 2015, 52, 1535-1547. [CrossRef]

2. Fialkow, A. Conformal geodesics. Trans. Am. Math. Soc. 1939, 45, 443-473. [CrossRef]

3. Ishihara, S. On infinitesimal concircular transformations. Kodai Math. Sem. Rep. 1960, 12, 45-56. [CrossRef]

4. Obata, M. Conformal transformations of Riemannian manifolds. J. Differ. Geom. 1970, 4, 311-333. [CrossRef]

5. Yano, K. Concircular geometry I. Concircular transformations. Proc. Imp. Acad. Tokyo 1940, 16, 195-200. [CrossRef]

6. Chen, B.-Y. A simple characterization of generalized Robertson-Walker space-times. Gen. Relativ. Gravit. 2014, 46, 1833. [CrossRef]

7. Duggal, K.L.; Sharma R. Symmetries of Spacetimes and Riemannian Manifolds; Springer-Science + Busisness Media B. V.: Berlin/Heidelberg, Germany, 1999.

8. Hawking, S.W.; Ellis, G.F.R. The Large Scale Structure of Space-Time; Cambridge University Press: Cambridge, UK, 1975.

9. Mantica, C.A.; Molinari, L.G. Generalized Robertson-Walker space times, a survey. Int. J. Geom. Methods Mod. Phys. 2017, 14, 1730001. [CrossRef]

10. Takeno, H. Concircular scalar field in spherically symmetric space-times I. Tensor 1967, 20, 167-176.

11. Yano, K. On torse forming direction in a Riemannian space. Proc. Imp. Acad. Tokyo 1944, 20, 340-345. [CrossRef]

12. Mantica, C.A.; Molinari, L.G. Twisted Lorentzian manifolds, a characterization with torse-forming time-like unit vectors. Gen. Relativ. Gravit. 2017, 49, 51. [CrossRef]

13. Marrero, J.C. The local structure of trans-Sasakian manifolds. Ann. Mat. Pura Appl. 1992, 162, 77-86. [CrossRef] 
14. Mihai, A.; Mihai, I. Torse forming vector fields and exterior concurrent vector fields on Riemannian manifolds and applications. J. Geom. Phys. 2013, 73, 200-208. [CrossRef]

15. Mikes, J.; Rachunek, L. Torse-Forming vector fields in T-symmetric Riemannian spaces, Steps in Differential Geometry. In Proceedings of the Colloquium on Differential Geometry, Debrecen, Hungary, 25-30 July 2000; pp. 219-229.

16. Chen B.-Y. Pseudo-Riemannian Geometry, d-Invariants and Applications; World Scientific: Hackensack, NJ, USA, 2011.

17. Deshmukh, S.; Turki, N.B.; Vîlcu, G.-E. A note on static spaces. Results Phys. 2021, 104519. [CrossRef]

18. Yano, K. Integral Formulas in Riemannian Geometry; Marcel Dekker: New York, NY, USA, 1970.

19. Chen, B.-Y. Rectifying submanifolds of Riemannian manifolds and torqued vector fields. Kragujev. J. Math. 2017, 41, 93-103.

20. Chen, B.-Y. Classification of torqed vector fields and its applications to Ricci solitons. Kragujev. J. Math. 2017, 41, 239-250. [CrossRef]

21. Deshmukh, S.; Turki, N.; Alodan, H. On the differential equation governing torqued vector fields on a Riemannian manifold. Symmetry 2020, 12, 1941. [CrossRef]

22. O'Neill, B. Semi-Riemannian Geometry with Applications to Relativity; Academic Press: London, UK, 1983.

23. Petersen, P. Riemannian Geometry; Graduate Texts in Mathematics-171; Springer: New York, NY, USA, 1997.

24. Shaikh, A.A.; Hui, S.K. On extended generalized $\phi$-recurrent $\beta$-Kenmotsu manifolds. Publ. de L'Inst. Math. New Ser. 2011, 89, 77-88.

25. Pigola, S.; Rimoldi, M.; Setti, A.G. Remarks on non-compact gradient Ricci solitons. Math. Z. 2011, 268, 777-790. [CrossRef]

26. Fischer A.E.; Marsden, J.E. Manifolds of Riemannian metrics with prescribed scalar curvature. Bull. Am. Math. Soc. 1974, 80, 479-484. [CrossRef] 